\title{
Muséologies
}

Les cahiers d'études supérieures

\section{Chantal Pontbriand, critique d'art et commissaire}

\section{Alessandra Mariani et Marie-Ève Courchesne}

Volume 3, numéro 1, automne 2008

URI : https://id.erudit.org/iderudit/1033580ar

DOI : https://doi.org/10.7202/1033580ar

Aller au sommaire du numéro

Éditeur(s)

Association Québécoise de Promotion des Recherches Étudiantes en Muséologie (AQPREM)

ISSN

1718-5181 (imprimé) 1929-7815 (numérique)

Découvrir la revue

Citer ce document

Mariani, A. \& Courchesne, M.-È. (2008). Chantal Pontbriand, critique d'art et commissaire. Muséologies, 3(1), 62-73. https://doi.org/10.7202/1033580ar d'utilisation que vous pouvez consulter en ligne.

https://apropos.erudit.org/fr/usagers/politique-dutilisation/ 
Dialogue deux

Chantal Pontbriand,

Critique d'art et commissaire

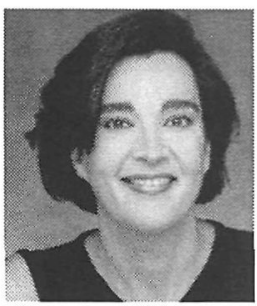

[réalisé par Alessandra Mariani avec la contribution de Marie-Ève Courchesne le 9 juillet 2008]

Critique d'art et commissaire, Chantal Pontbriand vit à Paris et à Montréal. Directrice-fondatrice de la revue d'art contemporain PARACHUTE, elle a été commissaire d'une vingtaine d'expositions, quinze événements internationaux, et douze colloques, principalement dans les champs de la performance, l'installation multimédias, la vidéo et la photographie. De 1982 à 2003, elle a dirigé le FIND (Festival international de nouvelle danse) à Montréal. Parmi ses publications, on compte Fragments critiques, Éditions Jacqueline Chambon, 1998, et Communauté et gestes, Éd. PARACHUTE, 2000. Elle travaille actuellement sur plusieurs livres dont Le grand labo, essai sur les pratiques artistiques contemporaines et l'institution et sur une exposition au Jeu de Paume : HFI RG [Harun Farocki / Rodney Graham]. 
ALESSANDRA MARIANI

Décrivez-nous brièvement ce qu'est I'IKT.

Chantal Pontbriand

L'IKT (International Association of Curators of Contemporary Art) est la plus importante association internationale de conservateurs en art contemporain. Il existe des associations locales de ce type (notamment en France, en Angleterre, et aux États-Unis), mais l'IKT joue un rôle international et représente près de 500 membres actifs dans 50 pays. L'organisation est en pleine expansion et le recrutement vabon train. À l'occasion du congrès qui vient d'avoir lieu au Canada, pour la première fois en dehors de l'Europe, nous avons recruté 30 nouveaux membres canadiens, ce qui fait passerle contingent canadien à 35. On peut comparer l'IKT au CIMAM (International Committee of ICOM for Museums and Collections for Modern Art), qui regroupe des directeurs, des conservateurs et des professionnels qui œuvrent surtout dans les musées d'art contemporain. L'IKT rallie ce même type de professionnels, mais elle accueille aussi des conservateurs indépendants de même que des directeurs de galeries universitaires, de centres d'art et de fondations vouées à l'art contemporain. L'IKT est donc à la base un organisme plus diversifié que le CIMAM. L'association a été fondée il y a plus de 40 ans, au même moment où émergeait la notion d'art contemporain comme domaine distinct. Ses membres sont en général très impliqués dans la recherche et le développement: cette dimension pragmatique propre à l'association est mise en évidence lors des congrès qui donnent préséance aux visites sur le terrain et aux échanges entre collègues. Les congrès fournissent l'occasion de partager les regards des différents membres qui témoignent d'une conception singulière et diversifiée du travail de conservateur. Quand on habite la Hongrie ou le Maroc, on ne travaille pas comme on le fait aux États-Unis ou en France. De plus, le programme des visites permet une recherche intensive sur le terrain : les directeurs ou les responsables des lieux que l'on visite, en l'occurrence tant des musées, des galeries universitaires, que des centres d'artistes et de production, exposent leurs programmes et leurs orientations. Cela permet de prendre contact avec des réalités différentes, une manière autre de faire les choses. La partie du congrès annuel consacrée au colloque, où l'on invite des spécialistes, aborde des sujets comme les droits d'auteurs, le format des expositions ou, cette année même, la relation art - économie.

Alessandra Mariani

Y a-t-il une publication liée à ces congrès?

Chantal Pontbriand

Malheureusement non, maisj'avoue que ce serait en effet une excellente idée que de matérialiser le contenu des échanges de cette façon. Lors des périodes d'échanges dans le cadre du congrès par exemple, les membres ont pu discuter des questions des conflits d'intérêt potentiels engendrés par le désistement de l'État et la présence 
croissante du secteur privé. Ce type de questionnement pourrait mener à des développements au-delà du congrès lui-même, des groupes de recherche peut-être. Avec davantage de moyens, il $y$ aurait en effet encore beaucoup à faire. Autrement, nous pourrions profiter de l'intérêt manifesté lors de ces rencontres pour demander aux membres d'approfondir leurs réflexions et de contribuer au développement du site Web de l'IKT par des textes ou des commentaires.

Pour le dernier congrès qui s'est tenu ici à Montréal, nous avons produit un guide des artistes locaux à l'intention des participants. Cet exercice - un petit bottin illustré de 80 artistes -, bien qu'embryonnaire, permettait aux visiteurs étrangers de se familiariser rapidement avec la scène montréalaise et d'en saisir le dynamisme particulier. C'était une première dans le contexte de l'IKT.

ALESSANDRA MARIANI

Qu'est-ce qu'un événement de cette envergure représente pour le Québec, et plus particulièrement pour Montréal, et ses institutions culturelles? Quelles en sont les répercussions?

Chantal Pontbriand

Je croyais beaucoup à la tenue du congrès au Québec parce que nous avons très peu de liens en arts visuels avec la scène internationale. Cette dernière n'a plusl'enthousiasme pourle Canada qui prévalait au cours des années 1980 , période pendant laquelle nous avons reçu un très grand nombre de visites de la part de conservateurs internationaux. Le Canada était alors très en vue étant donné qu'il produisait à cette époque des artistes particulièrement innovateurs dans le domaine des nouvelles technologies, de la photographie, de la vidéo et de la performance. Des grands noms en sont issus : Jeff Wall, Rodney Graham, Jana Sterbak, Geneviève Cadieux, etc. Parla suite, la dynamique de l'art contemporain s'est transformée et s'est élargie à tous les continents. L'Afrique et l'Asie sont aussi devenues des lieux intéressants pour l'art contemporain; la place du Canada en a proportionnellement perdu de l'importance. Il faut aujourd'hui multiplier les dispositifs pour assurer la place du Canada sur la scène internationale. En ce sens, la tenue $d u$ congrès annuel de l'IKT ici est pour moi un excellent moyen d'avoir de l'influence sur la scène internationale. Chacun des membres est un multiplicateur dans son milieu respectif:ce sont des gens qui ont de l'influence, des réseaux internationaux distincts et multiples, des publics diversifiés; ce sont des gens qui circulent, qui exposent les artistes et les idées auprès de multiples publics. En amont, au moins 500 personnes ont reçu au cours des douze mois précédant l'événement l'invitation à venir au Canada et à voir ce qui s'y fait. Le programme du congrès, particulièrement riche et diversifié, a certes contribué à cette participation, plus importante en nombre que celle de congrès précédents. On a pu se rendre compte de la richesse du milieu montréalais et québécois. Malgréle fait que nos 


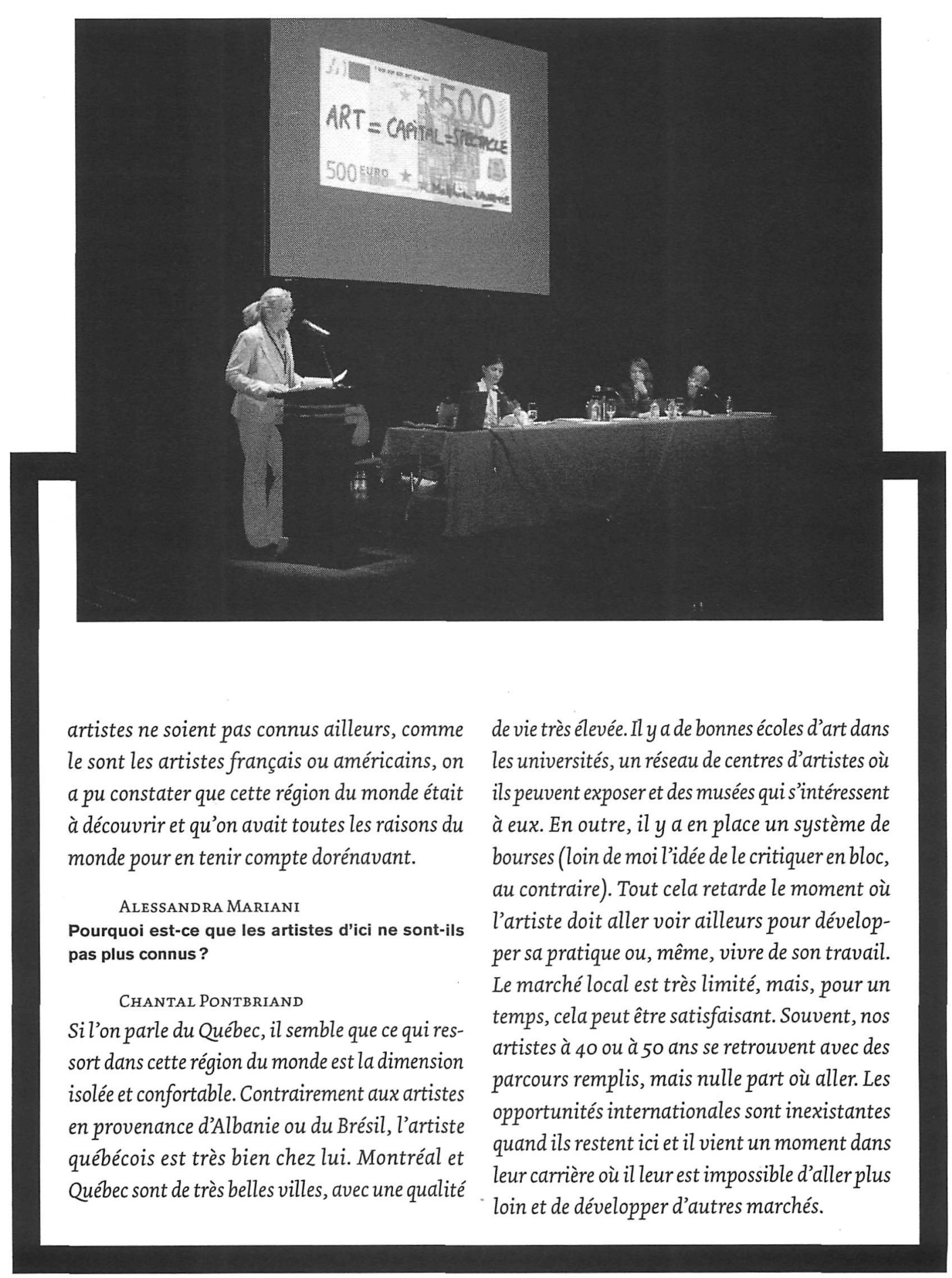


Nous sommes dans un système fermé sur lui-même. Il y a beaucoup à faire pour ouvrir nos frontières mentales et géographiques. Nous sommes par ailleurs très gâtés parle système qui a été mis en place et qui a été formidable jusqu'à récemment, parce qu'il a encouragé une certaine excellence et a permis le développement de pratiques très innovantes. Nous continuons donc de voir de très bons artistes émerger, mais nous ne voyons pas chezles artistes quebécois actuels cette urgence d'aller s'imposer à New York ou à Paris, de se faire connaître dans un monde elargi. C'est difficile pour ceux qui décident de le faire; ils doivent passer outre la notoriété qu'ils ont obtenue ici, en ayant bénéficié de bourses, de prix et de multiples possibilités d'expositions dans des lieux sans but lucratif.

Les artistes québécois et canadiens ne sont pas seulement confortés par ce système qui permet d'avancer rapidement alors qu'ils sont jeunes, il y a plus. Le fait de vivre dans une ville comme Montréal, par exemple, ne donne pas l'impression d'être privé de quoi ce soit : nous $y$ sommes très bien nourris culturellement à cause $d u$ foisonnement qui y existe, non seulement sur le plan des arts visuels, mais dans les secteurs de la musique, de la danse, du théatre, de l'architecture et du cinéma. Il faut ajouter les facteurs liés à la dimension économique qui ne favorisent pas notre environnement artistique. Parce que nous ne faisons pas partie de la scène internationale, parce que nous n'avons pas encore localement suffisamment de collectionneurs importants d'art international, les directeurs de galeries ou les collectionneurs d'autres pays ne viennent pas au Canada voir ce qui s'y fait. La dynamique de marché doit aller dans les deux sens, il s'agit tout simplement du principe de l'échange. Le jour où nous aurons des collectionneurs locaux importants qui s'intéressent non seulement à l'art canadien, mais aussi aux œuvres d'artistes d'autres pays, nous verrons les choses changer ici surle plan $d u$ marché pour nos artistes. Il est important que les gouvernements se penchent davantage sur cette question parce que c'est là qu'ils peuvent agir, en créant des incitatifs fiscaux, des politiques d'achats plus généreuses dont pourraient bénéficier autant les instances privées que publiques, et ce, toujours dans le but de créer une augmentation du commerce tant au niveau national qu'international.Je crois qu'il n'y a aucun risque à ce genre d'incitatif; au contraire, nous en avons grand besoin. En outre, cela aurait l'avantage de nous positionner un peu plus comme des gros joueurs sur la scène internationale et l'on ne pourrait plus nous ignorer. 
AlessandRa MARIANi

Et l'éducation dans cette équation? N'a-t-elle pas son rôle à jouer? En fait-on assez?

Chantal Pontbriand

Malheureusement, la diffusion qui est faite de ce côté par les médias de masse n'est pas celle qui est souhaitée parce qu'elle ne reflète pas ce qui se passe d'intéressant et de valable, ici ou même sur la scène internationale. Lorsqu'on nous présente l'art contemporain à la télévision, il s'agit très souvent de photos ou de tableaux faits par une célébrité déjà connue des médias. Cela est très problématique et reflète le point de vue timoré non seulement des médias, mais d'une certaine communautéde gens qui pourraient, parce qu'ils en ont les moyens, s'intéresser à l'art contemporain, mais qui n'osent pas. À New York, par exemple, on ose, parcequec'est un must; illefaut, sans quoi on ne s'inscrit pas dans une dynamique sociale en évolution. Il y a un sens général comme quoil'art contemporain est important, qu'on doit être au courant et s'y intéresser.

Comment créer ce sentiment ici? En proposant des visites d'expositions dès l'age scolaire et en valorisant l'enseignement de l'art. Au-delà de cela, les médias doivent jouer le rôle qu'on attend d'eux surle plan artistique autant que surle plan de la politique, de l'économie ou du sport. Les musées doivent eux aussi utiliser davantage leur intelligence pour offrir des programmations qui font sens sur une longue durée et "construire" des publics avertis et critiques. Là aussi, il y beaucoup à faire.
ALESSANDRA MARIANI

Pourquoi avez-vous tenu à ce que l'événement se tienne à Montréal et à Québec?

Chantal Pontbriand

Je savais qu'il s'agissait d'une chance extraordinaire de recevoir chez nous un grand nombre de conservateurs en art contemporain en provenance de nombreux pays et d'attirer l'attention surce qui se fait ici plutôt qu'ailleurs. Il $y$ a des centaines de studios à Montréal, les congressistes ont pu le constater lorsque nous les avons emmenés à la Galerie Clark. Cette visite coïncidait avec un événement appelé open studios, qui permettait aux intéressés de visiter les galeries et les studios du quartier du Mile End et de ses environs. Nous avons fait coïncider le congrès avec le vernissage de la nouvelle Triennale du Musée d'art contemporain où 40 artistes étaient exposés. Il y avait aussi une très belle exposition - Intrus - au Musée des beaux-arts du Quebec qui mêlait une vingtaine d'artistes contemporains québécois aux collections d'œuvres faisant état de l'histoire de l'art au Québec. Lorsqu'on a la chance de voir tout ça, on ne peut s'empêcher de constater qu'il y a beaucoup d'artistes de bon calibre qui travaillent ici. Un grand nombre parmi les 150 congressistes ouvriront certainement l'œil lorsqu'ils rencontreront des œuvres des artistes découverts ici. Aussi, certains reviendront, j'en suis certaine.

Évidemment, il s'agit d'un événement de quelques jours qui n'est pas appelé à se répé- 
ter. Si nous ne trouvons pas d'autres dispositifs pour attirer le milieu international, et si nos artistes ne se mettent pas à bouger davantage, cela n'aura peut-être été au bout du compte d'un coup d'épée dans l'eau. Il y a bien sûr des organismes qui travaillent à créer des ponts internationaux en arts visuels, mais très souvent à petite échelle; c'est le cas, en autres, des résidences d'artistes. Celles-ci peuvent aider, mais elles ne sont pas une fin en soi et leur efficacité est limitée si l'artiste ne fait pas d'efforts pour se faire connaître, dans le pays d'accueil et ailleurs. Je pense qu'il est essentiel de produire des manifestations d'envergure internationale au Canada afin d'attirer l'attention de façon périodique et qu'il ne faut pas seulement se concentrer sur les artistes locaux. Je suis convaincue du fait qu'il est plus profitable de réaliser des expositions qui mêlent des artistes locaux et internationaux dans un contexte suffisamment bien garni financièrement, ce qui n'a pas étéle cas jusqu'à maintenant. La seule biennale que nous ayons, celle de Montréal, manque désespérément de fonds et de lieux adéquats. Le budget allouéà cette activité n'équivaut pas à celui de la Biennale d'Istanbul, par exemple. Ensuite, il faut vraiment jouerdans l'excellence, d'autant plus que nous sommes un petit pays sur la scène de l'art contemporain. Il faut être encore plus pointu, plus habile et plus intelligent. La tendance actuelle dans notre pays est plutôt d'être ultra-démocratique, d'inclure beaucoup de joueurs, defaire participer beaucoup d'instances locales, et ce ne sont pas nécessairement ces façons de faire qui produisent les meilleurs résultats. Par-dessus tout, il nous faut viser plus haut, créer des têtes d'affiches, des héros, des vedettes, en élevant nos critères. C'est ce dont nous avons besoin pour aller à la rencontre de la planète.

L'IKT tient des congrès régulièrement depuis une douzaine d'années. C'est d'autant plus extraordinaire que nous ayons réussi à en obtenir un au Canada. Nous avons reçu $80 \%$ des voix pour la tenue du congrès à Montréal, alors qu'on devait convaincre les membres de l'association de se déplacer pour la première fois en dehors de l'Europe, où réside la majorité. Les prochains congrès auront lieu à Helsinki en 2009, à Athènes en 2010 et à Luxembourg en 2011. J'espère que les Canadiens s'y rendront en grand nombre pour nous permettre de continuer ce que nous avons commencé cette année en termes de reconnaissance et de participation à la scène internationale.

ALESSANDRA MARIANI

Quelles tactiques et quelles stratégies sont nécessaires pour planifier cet événement?

\section{Chantal Pontbriand}

Pour obtenir la venue d'un événement comme celui de l'IKT, il faut faire preuve de sa capacité à accueillir une structure internationale comme celle-ci surles plans organisationnel et financier et démontrer l'intérêt du programme que l'on 

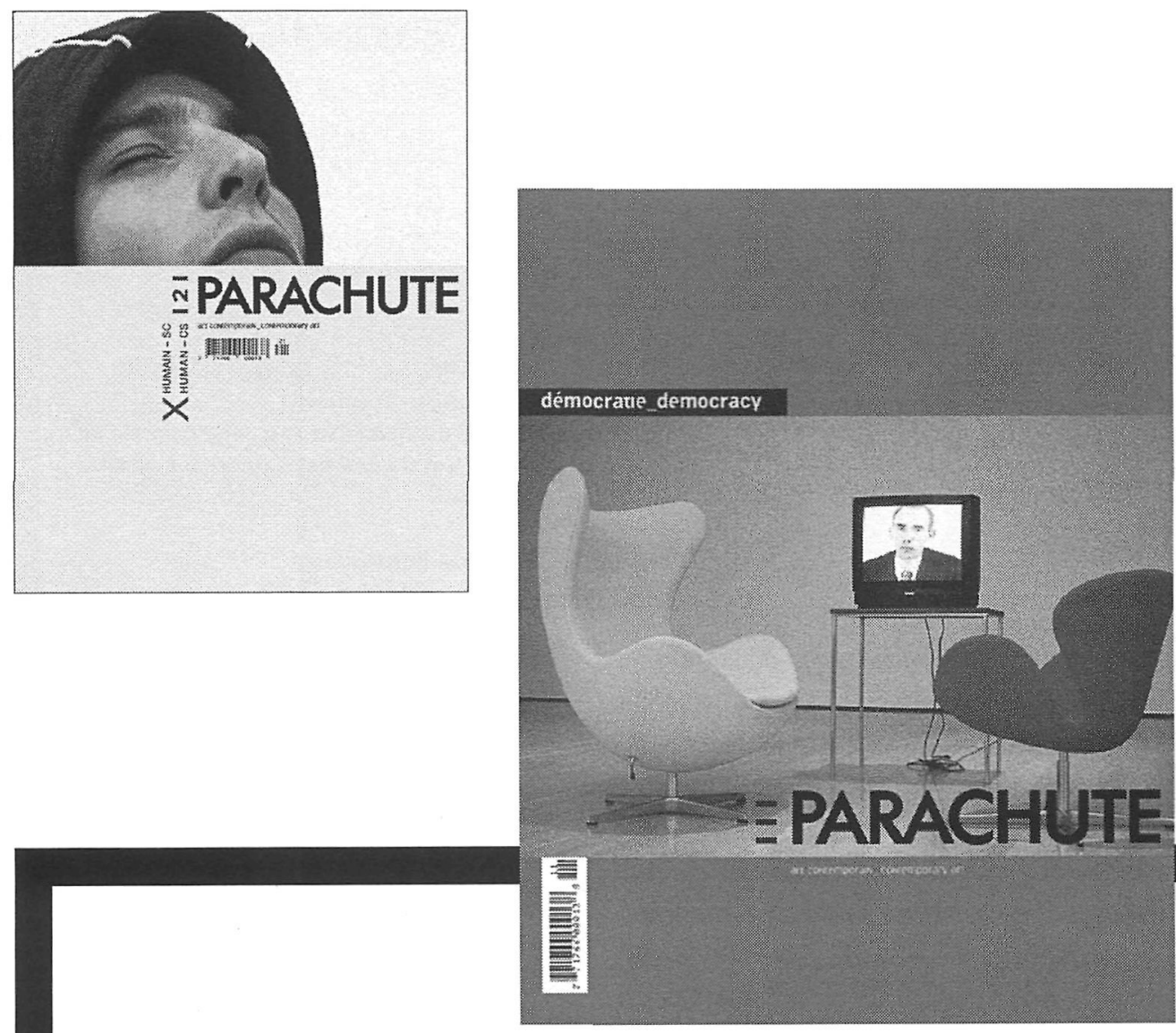

propose. Dans notre cas, le programme n'a pas été particulièrement difficile à élaborer, parce que je savais pertinemment qu'il y avait de bons musées à voir, de même que d'excellents centres d'artistes et lieux de production. Il faut aussi souligner la taille de la communauté artistique de Montréal : ce ne sont pas toutes les villes qui sont visitées par l'IKT qui ont la chance d'avoir autant d'artistes qui y travaillent. Nous avons inclus dans le programme, c'était aussi une première pour un congrès de l'IKT, une grande fête réunissant 80 artistes de Montréal et les 150 membres présents. La notion de rencontre et d'hospitalité est à la base de mes préoccupations depuis longtemps. Que ce soit avec la revue d'art contemporain PARACHUTE ou le Festival international de nouvelle danse (FIND), quej'ai dirigé pendant 20 ans, l'idée était de susciter des croisements de culture et d'attirer l'attention surles artistes d'ici à l'échelle internationale et vice versa, de faire connaître ici les principaux acteurs de cette scène étargie.

Avec un tel objectif en vue, il est impératif de se munir d'une programmation extrêmement réfléchie et axée surl'excellence : il faut, à chaque 
fois, un équilibre activement recherché des forces entre ce qui se fait ici et ce qui se produit ailleurs. Dans le domaine des arts visuels en général, il y a ici fort à faire en ce sens. Je suis convaincue que si je pouvais appliquer ces stratégies d'hospitalité au milieu de l'art visuel, j'arriverais à mes fins. PARACHUTE nous a bien servis en tant qu'outil de développement, mais une revue n'a pas la force immédiate de l'événement ou l'autorité quel'on confere habituellement à une institution de type muséal. Son pouvoir d'action est à long terme, alors que son impact, bien que potentiellement profond, est moins visible. Un événement permet aux acteurs de la scène internationale et aux leaders d'opinion de voir ce qui se fait en temps réel. Il crée un public elargi, il conscientise les médias. Une revue comme PARACHUTE, même tirée à 5000 exemplaires et distribuée dans plus de 40 pays, ne peut avoir le même impact à court terme qu'un événement. Avec le FIND, nous avons réussi à mettre la danse montréalaise sur la carte du monde grace à des stratégies d'excellence dans la programmation, à un équilibre sans compromis entre la production locale et internationale, à des stratégies visant à établir des liens personnalisés et des réseaux l'internationaux; c'est en raison de la précision de nos objectifs et de la qualité de leur mise en œuvre que nous nous sommes démarqués en tant qu'événement et que notre entourage en a bénéficié.

\section{Alessandra Mariani}

Quel est l'état du marché de l'art contemporain au Québec? Où est-il situé par rapport aux circuits internationaux?

\section{Chantal Pontbriand}

C'est un marché en développement, surtout concerné parl'achat d'art québécois : il y a à cela bien sûr des exceptions. Reste que très peu de galeries s'occupent d'art contemporain surle plan commercial. Il n'y a pas actuellement de galerie de calibre « international » qui présente des artistes d'ailleurs et des artistes d'ici de façon régulière et la raison en est simple : nous n'avons pas de clientèle qui soit prête à en payer le prix. C'est une situation que je qualifierais d'épineuse, qui n'a pas changédepuis des années maintenant et qui est d'autant plus difficile à comprendre dans un contexte mondial où l'art contemporain est en croissance exponentielle.

\section{ALESSANDRA MARIAN}

Quelle en est la raison?

\section{Chantal Pontbriand}

Il s'agit encore du fait insulaire : les gens d'ici ne se tournent simplement pas facilement vers l'art actuel. Il y a maintenant en Asie et en Inde de plus en plus de personnes qui font le choix d'investir dans l'art contemporain. Elles désirent faire partie de cette dynamique internationale et savent très bien qu'il est dans leur intérêt d'être associé à l'art contemporain et de posséder une photographie d'Andreas Gursky ou de Cindy Sherman! L'art contemporain n'échappe pas à 
ce goût qui se développe avec la connaissance : plus on connaît, plus on devient passionné et plus cela devient une exigence ou, à tout le moins, un must. Alors, lorsque vous me demandez où se situe le marché de l'art contemporain au Québec, que puis-je vous répondre? Au niveau zéro! Il n'y a pas de liens possibles à faire avec ce qui se passe sur le marché mondial : des efforts incroyables sont faits par certaines galeries, mais elles-mêmes vous diront qu'il faut des énergies considérables pour simplement obtenir une place dans des foires internationales. Participer à ces activités demeure encore le seul moyen d'ameliorer la situation, parce que cela donne un peu de visibilité. Les choses peuvent toujours basculer à force de faire des efforts, comme nous le prouve la chute du mur de Berlin : personne n'avait prédit le cours des événements. Un jour, les choses changeront, mais seulement à force d'efforts continus.

AlESSANDRA MARIANI

Comment l'IKT permet-elle de tisser des liens entre les institutions locales et internationales?

\section{Chantal Pontbriand}

L'IKT est convaincue que le meilleur moyen de créer des liens est de voir les lieux, de se rendre compte sur place de ce que ces lieux ont comme objectifs, de faire la connaissance de ceux qui montent et pensent les expositions. Cette démarche permet de repérer les collègues qui travaillent dans une même optique et d'entrevoir des projets communs.
ALESSANDRA MARIANI

Quelle est la couverture médiatique de notre scène artistique en Europe ? Est-ce un marché bien établi ou est-il encore à construire?

\section{Chantal Pontbriand}

Elle est, je dirais, presque inexistante. Nos artistes ne sont pas connus. Quand on vient à Montréal, on ne sait pas àl'avance le nom des artistes qui y travaillent. C'est pourquoi nous avons dû, pour aider nos collègues à s'y repérer, créer un petit guide avec un minimum de 80 artistes, le Short Guide: Montreal Art Atlas. Tout est à construire, comme nous l'avons fait pour la danse avec le FIND pendant 20 ans, avec la collaboration des artistes. Ce genre d'événement nous a permis de projeter sur la scène locale et internationale les Édouard Lock, Marie Chouinard et autres. Il faut elargir nos horizons, créer un l'impact et tous en profiteront.

\section{ALESSANDRA MARIANI}

Est-ce que les départements des communications de nos institutions culturelles sont bien outillés pour faire la promotion de notre marché?

\section{Chantal Pontbriand}

Non et ce n'est pas nécessairement leur rôle, c'est un rôle davantage lié aux événements. Cependant, les musées peuvent fortement agir sur la reconnaissance des artistes et la compréhension générale de l'art contemporain, ce qui influence certainement le marché. Cela se fait tant parl'excellence et la cohérence de la programmation que par la vision dont ils font preuve. Un programme peut être très bon, 
c'est-à-dire qu'il peut être constitué de choix pertinents, mais il sera plus ou moins efficace s'il y manque une vision d'ensemble, un projet intellectuel et artistique fort à la base. Je pense au Musée d'art contemporain de Barcelone (MACBA), qui est pour moi une référence en ce sens.

Alessandra Mariani

Nos médias, tous types confondus (journaux, radio, télévision, publications, etc.), couvrent-ils suffisamment les événements comme I'IKT et discutent-ils suffisamment des artistes contemporains?

\section{Chantal Pontbriand}

Le congrès de IKT était difficile à couvrir parce qu'il s'agissait d'un événement privé, exclusif aux membres. Même s'il s'agissait en même temps d'un événement d'intérêt public parce qu'il a un impact direct sur le développement et la reconnaissance du milieu local, nous ne nous attendions pas à la couverture médiatique que nous avons reçue. En plus d'articles dans les revues spécialisées Ciel Variable et CMagazine, il y a eu un texte dans Le Devoir et un passage à l'émission de Christiane Charette. Il y a encore chez nous une très grande timidité envers l'art contemporain, ce qui ne nous sert pas; celui-ci est toujours perçu comme déstabilisant. S'il était possible d'envoyer 50 journalistes et recherchistes québécois à la foire de Miami ou à la Biennale d'Istanbul, un plus large public, j'en suis convaincue, parviendrait finalement à comprendre toute l'énergie et la puissance qui émanent de ces événements, toute leur importance pour saisir notre contemporanéité et faire partie d'un monde plusgrand.

\section{AlessandRa MaRIanI}

Comment la presse écrite spécialisée en art contemporain influence-t-elle la façon dont les musées produisent des expositions, et vice versa?

\section{Chantal Pontbriand}

C'est quelque chose de très difficile à évaluer. La presse spécialisée attire l'attention sur ce qui est réellement important dans le domaine, ce qui mérite davantage d'attention et d'intérêt de la part du milieu artistique et du public elargi. Une revue se démarque par la qualité de ses choix à long terme et sur la pertinence et la rigueur de ses analyses. On ne peut s'empêcher de penser que les choix que nos musées locaux font ont été influencés par PARACHUTE et par d'autres publications de référence. On peut aussi penser que s'il n'y avait pas eu ici de revue qui ait fait le choix de s'investir spécifiquement dans la création d'une dynamique internationale, nos musées locaux seraient moins ouverts à cette même dynamique. Nous avons ainsi travaillé à elargir notre champ de vision collectif. 
ALESSANDRA MARIANI

Comment les publications spécialisées en art contemporain peuvent-elles conquérir une part de l'espace médiatique? Comment sensibiliser davantage différents publics?

\section{Chantal Pontbriand}

Il s'agit de deux mondes parallèles, les médias de l'industrie du spectacle et nous, bien qu'il soit évident que les critiques dans les journaux lisent les revues spécialisées, plus queles recherchistes à la telévision.J'ai toujours pensé qu'il aurait pu $y$ avoir un sas entre Le Devoir et PARACHUTE, par exemple, et qu'on aurait pu établir une collaboration particulière, mais cela ne s'est pas produit. J'ai aussi quelquefois réfléchi à l'idée d'une version telévisuelle ou radiophonique de PARACHUTE. L'Internet n'est pas encore suffisamment puissant pour le commun des mortels qui s'intéresse à des questions spécialisées. Je le dis tout en sachant que le site Web de PARACHUTE, alors que l'on publiait encore la revue, attirait plus d'internautes que celui du Palais de Tokyo à Paris!

\section{Alessand Ra MARIANI}

Comment entrevoyez-vous l'avenir de l'art contemporain au Québec depuis que le congrès de l'IKT s'est tenu ici?

\section{Chantal Pontbriand}

L'environnement de l'art contemporain n'est pas pressé, il ressemble à celui de la situation politique au Québec. On fait présentement chez nous beaucoup de gestion, sans grand idéal. En haut des priorités, il y a la question de la santé, ce qui est facile à comprendre parce qu'elle touche tout le monde et qu'elle est au fond matérielle : les questions culturelles ne sont pas prioritaires. Quelquefois, le debat collectif touche à des questions culturelles. Ce fut le cas de la récente commission sur les accommodements raisonnables, un exercice intelligent et nécessaire.J'aurais souhaité qu'il fût lié aux pratiques artistiques qui ont cours et qui touchent ces questions essentielles de notre temps. C'est encore heureux que cela soit possible de mener une entreprise de cette nature au Québec : dans de nombreux pays, on n'ose pas le faire ouvertement; cela démontre qu'il y a ici même un fort potentiel d'ouverture.

Je crois que la situation de l'art contemporain pourrait s'ameliorer si une plus grande volonté politique se manifestait à son égard. À cela, ajoutons qu'il faut aussi que le milieu même de l'art contemporain fasse les choix de se départir de sa situation confortable, de demander davantage, d'oser plus, de s'aventurer plus loin. Il faut qu'une sorte de révolte, moteur de changement, fasse jour; c'est de la révolte que naissent les révolutions. 